\title{
Review: individual applied relaxation and cognitive behavioural therapy are effective psychological treatments for generalised anxiety disorder
}

Fisher PL, Durham RC. Recovery rates in generalized anxiety disorder following psychological therapy: an analysis of clinically significant change in the STAI-T across outcome studies since 1990. Psychol Med 1999 Nov;29:1425-34.

QUESTION: In adult patients with generalised anxiety disorder (GAD), what are the treatment effects of psychological therapies when using standardised Jacobson criteria for evaluating scores on the State-Trait Anxiety Inventory (STAI-T)?

\section{Data sources}

Studies were identified by searching Medline, PsycLIT, and the Cochrane Controlled Trials Register using the keywords generalis(z)ed anxiety disorder, treatment outcome, psychological trials, efficacy study, comparative study, and clinical trial. The search period covered January 1987 to December 1998. Bibliographies of identified studies were scanned and researchers in the field were contacted.

\section{Study selection}

Studies were selected if there was random assignment of patients (between 18-65 years of age) with GAD (diagnosed using a structured diagnostic interview according to DSM-III-R or DSM-IV criteria) to $\geq 2$ psychological treatments or control conditions.

\section{Data extraction}

Individual patient data were obtained from the authors of each study on Spielberger STAI-T scores at baseline, after treatment, and at 6 months of follow up. Jacobson methodology for defining clinically significant change (in this case an 8 point difference on the STAI-T was required for indicating a reliable change) was used to allocate each patient to 1 of 4 outcomes; worse, unchanged, improved, or recovered. Data were aggregated across types of treatment.

Scottish Hist's Offic Scottish Home and Health Department, Edinburgh.

For correspondence: Dr R C Durham,

University of Dundee. Department of

Psychiatry, Ninewells

Hospital and Medical

School, Dundee,

Scotland DD1 9SY,

UK. Fax $+44(0) 138$

2633923.

\section{Main results}

6 studies met the selection criteria. 1 study was excluded because of a high dropout rate. The table summarises recovery rates by treatment approach at 6 months of follow up. Overall recovery rates using aggregated data from the 5 studies suggest that about $60 \%$ of patients make some form of improvement after psychological treatment but, on average, slightly $<40 \%$ can be consid-

Recovery rates by treatment approach at 6 months of follow up

\begin{tabular}{lllll} 
Treatment & N & $\begin{array}{l}\text { Recovery status } \\
\text { maintained from } \\
\text { post-treatment }\end{array}$ & $\begin{array}{l}\text { Recovery } \\
\text { status } \\
\text { achieved }\end{array}$ & $\begin{array}{l}\text { Overall } \\
\text { recovery } \\
\text { rate }\end{array}$ \\
\hline Individual AR & 38 & $52 \%$ & $8 \%$ & $60 \%$ \\
\hline Individual CBT & 87 & $41 \%$ & $10 \%$ & $51 \%$ \\
\hline Non-directive therapy & 16 & $19 \%$ & $19 \%$ & $38 \%$ \\
\hline Group CBT & 40 & $18 \%$ & $15 \%$ & $33 \%$ \\
\hline Group BT & 26 & $12 \%$ & $19 \%$ & $31 \%$ \\
\hline Individual BT & 28 & $7 \%$ & $4 \%$ & $11 \%$ \\
\hline Analytical psychotherapy & 23 & $0 \%$ & $4 \%$ & $4 \%$ \\
\hline
\end{tabular}

$\mathrm{AR}=$ applied relaxation; $\mathrm{CBT}=$ cognitive behavioural therapy; $\mathrm{BT}=$ behaviour therapy.

ered to be recovered in terms of STAI-T scores. 2 treatment approaches, individual cognitive behavioural therapy and applied relaxation, led to the highest overall recovery rates $(50-60 \%)$ at 6 months of follow up.

\section{Conclusion}

Individual applied relaxation and cognitive behavioural therapy are effective psychological treatments for generalised anxiety disorder when evaluated using scores on the State-Trait Anxiety Inventory.

\section{COMMENTARY}

An ongoing and vexing problem for clinical researchers in mental health is the issue of consolidating research findings obtained from different studies evaluating varying treatments for a particular disorder. At present, the best method for combining studies is meta-analysis. Meta-analyses usually combine odds ratios or effect sizes, neither of which give the clinician a good sense of how clinically important the difference between the groups actually is. Recently, a third measure has been introduced. This third approach to integrating the findings of various randomised controlled trials (RCTs) is known as computing a Reliable Change Index (RCI). RCIs require a common outcome measure, and the authors found a total of 6 RCTs evaluating psychological treatments for GAD that used as a common outcome measure scores on the STAI, the "gold standard" for measuring the chronic type of pervasive anxiousness associated with GAD. Computing the RCI and using the Jacobson methodology for defining clinically significant change enabled the reviewers to draw 1 of 4 conclusions: the number and proportion of clients who (1) got worse, (2) did not change, (3) got better, and (4) improved to within normal limits. Then the proportions of clients in each study falling into these 4 categories can be aggregated to arrive at overall conclusions based on the entire sample of individuals studied across all the studies. Obviously, clinicians and patients will find these types of conclusions ("How many improved?" "How many were cured?") far more comprehensible than interpreting an analysis of variance or figuring out the practical meaning of an effect size. Apart from the authors' supportable conclusions that CBT and relaxation training are moderately effective and reliable psychosocial treatments, this article is notable for exemplifying the application of the RCI and Jacobson methodology, which should become a required additional feature of statistical reportage for articles in the field of evidence-based mental health.

Bruce A Thyer, $\mathrm{PhD}$ University of Georgia Athens, Georgia, USA 